\title{
Les attentes éducatives des familles pauvres au Maroc
}

Une investigation empirique

Educational expectations of poor families in Morocco. An empirical investigation Las expectativas educativas de las familias pobres en Marruecos. Una investigación empírica

\section{Aomar Ibourk}

\section{OpenEdition}

\section{Journals}

Édition électronique

URL : https://journals.openedition.org/ries/3142

DOI : $10.4000 /$ ries.3142

ISSN : 2261-4265

Éditeur

France Education international

Édition imprimée

Date de publication : 1 avril 2013

Pagination : 119-124

ISBN : 978-2-85420-599-2

ISSN : 1254-4590

Référence électronique

Aomar Ibourk, "Les attentes éducatives des familles pauvres au Maroc », Revue internationale

d'éducation de Sèvres [En ligne], 62 I avril 2013, mis en ligne le 01 avril 2015, consulté le 05 juillet 2021 URL : http://journals.openedition.org/ries/3142 ; DOI : https://doi.org/10.4000/ries.3142 


\title{
Les attentes éducatives des familles pauvres au Maroc
}

Une investigation empirique

\author{
Aomar Ibourk
}

\section{INVESTISSEMENT EN ÉDUCATION ET ATTENTES PARENTALES}

Considérée comme un facteur déterminant de la réussite sociale et professionnelle de l'individu, la scolarité requiert de plus en plus une implication importante des familles marocaines qui visent la réussite éducative et sociale de leurs enfants. Cet engagement parental retient l'attention des chercheurs et des décideurs politiques soucieux de comprendre les facteurs socioéconomiques déterminants de ce phénomène et de prendre les mesures gouvernementales susceptibles de favoriser l'implication des parents dans l'éducation.

Idéalement, les parents devraient éprouver des aspirations élevées en matière d'éducation, en vue d'assurer un avenir prospère à leurs enfants. C'est ce qui est communément appelé l'altruisme intergénérationnel, lequel est tributaire du revenu mais aussi, et surtout, de l'image que se forge la famille de l'éducation. Rappelons ici que le marché de l'éducation présente une certaine particularité. En effet, ceux qui demandent l'éducation ne sont pas ceux qui la consomment. Les premiers sont les parents tandis que les seconds sont leurs enfants. Ainsi, les parents n'investiront dans l'éducation de leurs enfants que s'ils font preuve d'un réel engagement. Il existe une série de facteurs pouvant déterminer un tel engagement. La situation familiale des parents, leur revenu et leur niveau scolaire sont autant de facteurs favorisant l'investissement dans l'éducation des enfants. De nombreuses études ont montré l'influence positive de l'éducation des parents sur celle des enfants. Plus les parents sont scolarisés, plus ils ont tendance à inscrire leurs enfants plus longtemps à l'école, en raison de la valeur et de l'intérêt qu'ils accordent à l'école. Cela se traduit par des encouragements à l'étude, des conseils et du soutien scolaire, des exemples de réussite professionnelle, un comportement intellectuel et des moyens financiers mis à la disposition des enfants. De même, l'investissement parental en éducation dépend également de la profession des parents et de leur milieu social.

Par ailleurs, dans un contexte de démocratisation de l'école ainsi que d'une conjoncture socio-économique morose, deux analyses, aux antipodes l'une de l'autre, peuvent être développées en ce qui concerne les attentes éducatives des familles marocaines. La première souligne une prise de conscience 
grandissante des classes populaires de l'importance des études, dépeintes comme un levier incontournable de la promotion et de l'ascension sociales. Dans une telle optique, l'école peut se présenter comme un lieu de report de toutes les aspirations déçues de la classe ouvrière, les aspirations scolaires et professionnelles étant indissociables et souvent liées à une volonté d'ascension sociale. L'autre analyse est plus pessimiste. Elle relève un manque d'altruisme intergénérationnel dû à une absence totale d'appréciation des bienfaits de l'éducation. Cette analyse prend tout son sens dans un pays en développement comme le Maroc, qui présente des degrés de pauvreté et d'analphabétisme assez élevés. Sur un marché dont les agents sont mal-informés des bienfaits de l'éducation, le revenu immédiat lié au travail de l'enfant peut, vraisemblablement, représenter une part vitale dans le revenu de la famille.

Quelle est l'analyse qui permet de cerner au mieux les attentes éducatives des familles marocaines et plus précisément celles des plus pauvres ? L'objet de notre recherche est de tester s'il existe réellement des attentes éducatives croissantes de la part des familles pauvres au Maroc. Pour ce faire, une investigation empirique est menée dans une région caractérisée par l'expérimentation d'un programme de subvention conditionnée et qui affiche un taux de pauvreté élevé (une condition sine qua non du programme).

\section{UNE INVESTIGATION EMPIRIQUE}

\section{Le programme Tayssir}

Faisant partie du programme d'urgence du ministère de l'éducation nationale, Tayssir est un programme de transferts monétaires conditionnels qui apporte une contribution financière à des familles pauvres, à condition que leurs enfants utilisent le service d'éducation dispensé par l'école publique. Le but est d'agir sur l'abandon scolaire en neutralisant certains des facteurs qui réduisent la demande pour l'éducation, tels que les coûts de scolarisation directs ou indirects. Ce programme s'inspire de certaines expériences réussies dans d'autres pays, telles que Oportunidades au Mexique et Bolsa Familia au Brésil. L'aide est soumise à des conditions d'assiduité prédéfinies (moins de quatre absences par mois au cycle primaire et moins de six séances au cycle collégial). Pour le cycle primaire, les montants des subventions sont de : 60 dirhams marocains pour les élèves inscrits en première et deuxième années ; 80 dirhams marocains pour les élèves inscrits en troisième et quatrième années; 100 dirhams marocains pour les élèves en cinquième et sixième années. Pour le cycle collégial, la subvention est fixée à 140 dirhams marocains. Les subventions sont accordées sur dix mois, avec un versement bimestriel, à un parent désigné, soit les pères, soit les mères. Par ailleurs, si une école est choisie pour faire partie du programme, tous ses élèves doivent bénéficier des subventions, sous réserves de respect des conditions d'assiduité prédéfinies. 


\section{Méthodologie de l'enquête}

L'enquête s'est déroulée dans six communes rurales de la province de Kelaa des Serghna, dont cinq communes bénéficiaires de la subvention de Tayssir et une non bénéficiaire du programme. Le questionnaire préalablement établi a été rempli par des enquêteurs qui interrogeaient les parents d'élèves dans les écoles subventionnées et les écoles non subventionnées.

L'échantillon a été tiré d'une manière aléatoire à partir d'une base de données des ménages qui ont bénéficié du programme Tayssir. Afin de mesurer l'impact du programme, deux groupes ont été définis, l'un de cinq cents ménages avec 724 enfants bénéficiaires du programme et l'autre de 143 ménages avec 179 enfants non bénéficiaires du programme. L'administration du questionnaire a été effectuée dans un délai de trente jours.

Tableau 1. Niveau des parents et niveau d'aspiration en éducation

\begin{tabular}{|c|c|c|c|c|}
\hline & & \multicolumn{3}{|c|}{$\begin{array}{l}\text { Jusqu'à quel niveau continuerez-vous d'envoyer les enfants } \\
\text { à l'école, si l'État arrête de vous accorder la subvention? }\end{array}$} \\
\hline & & Primaire & Secondaire & Supérieur \\
\hline \multirow{4}{*}{$\begin{array}{l}\text { Niveau } \\
\text { d'instruction } \\
\text { du chef de ménage }\end{array}$} & Sans & $5,2 \%$ & $43,0 \%$ & $51,9 \%$ \\
\hline & Primaire & $3,5 \%$ & $36,1 \%$ & $60,4 \%$ \\
\hline & Secondaire & $4,6 \%$ & $43,1 \%$ & $52,3 \%$ \\
\hline & Supérieur & & $66,7 \%$ & $33,3 \%$ \\
\hline
\end{tabular}

Quel que soit leur niveau d'instruction, les parents demeurent fermes en ce qui concerne leur engagement pour l'éducation des enfants, même en cas d'arrêt des subventions. En ce sens, la volonté d'offrir aux enfants les moyens de parer à la vulnérabilité sociale à travers les études constitue un objectif ultime pour lequel les parents sont prêts à faire des sacrifices lourds et à subir davantage les conséquences de la pauvreté.

Aucune différence n'est constatée entre les parents à niveau d'instruction élevé et ceux dont le niveau d'instruction est faible. Ainsi, 60,4\% des parents à faible niveau d'instruction affichent des aspirations ambitieuses quant à la poursuite d'études de leurs enfants. Rares sont ceux qui envisagent pour eux un parcours court: ils ne sont que 5,2\% pour les parents sans aucun niveau d'éducation et $3,5 \%$ pour les parents ayant un niveau d'instruction primaire.

Ceci va dans le sens des hypothèses selon lesquelles les parents dont le niveau d'instruction est faible s'alignent sur ceux dont le niveau d'instruction est élevé, et accordent une valorisation et une importance aux études. 
Tableau 2. Engagement parental en cas d'arrêt des subventions

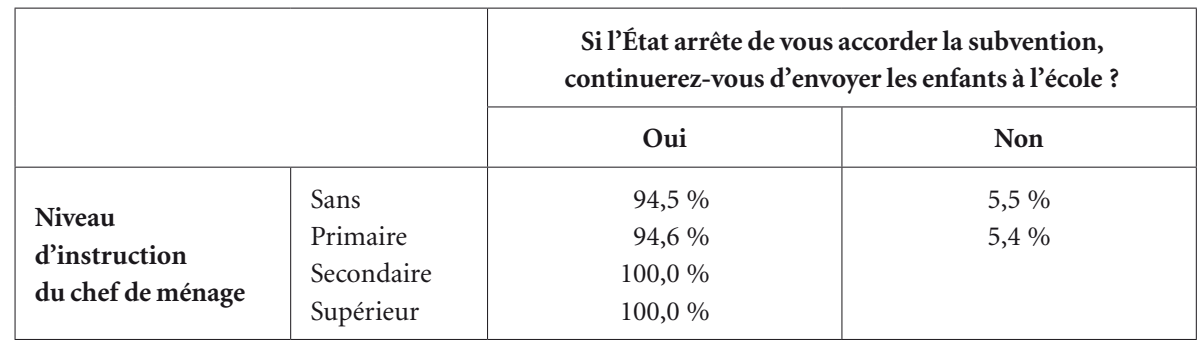

La prolongation des études des enfants est l'un des objectifs majeurs des parents. L’espoir d'éviter la précarité et de rattraper les modèles qui prévalent dans les classes dominantes place les parents dans l'obligation d'investir dans l'éducation prolongée des enfants, malgré l'incertitude quant aux débouchés des études supérieures.

La subvention n'est qu'un moyen qui permet de prendre en charge une partie des sommes investies dans l'éducation des enfants. Certes, le prolongement des études des enfants entraîne des charges financières lourdes, qui demandent plus de sacrifices, mais la volonté des parents d'offrir des conditions meilleures aux enfants et d'éviter les désavantages sociaux vécus prime, dans leurs déclarations, sur les difficultés prévues.

Tableau 3. Genres et attitudes des parents vis-à-vis des modalités de subventions

\begin{tabular}{|l|l|c|c|}
\hline \multicolumn{2}{|l|}{} & \multicolumn{2}{c|}{$\begin{array}{c}\text { Pensez-vous que les modalités de la subvention actuelle } \\
\text { encouragent les parents à envoyer les enfants à l'école ? }\end{array}$} \\
\cline { 3 - 4 } & Oui & Non \\
\hline Receveur & Père & $56,9 \%$ & $43,1 \%$ \\
de la subvention & Mère & $71,6 \%$ & $28,4 \%$ \\
\hline
\end{tabular}

Plus la femme reçoit la subvention, plus elle investit dans l'éducation et plus elle est encouragée à s'engager dans les études de ses enfants. Lorsque c'est la femme qui reçoit le montant de la subvention, ce dernier est utilisé directement pour l'éducation des enfants et constitue pour la femme une motivation réelle pour persister encore, afin d'offrir aux enfants de plus grandes chances d'un avenir meilleur. Les aspirations des mères se démarquent de celles des pères en ce qui concerne l'éducation des enfants. En d'autres termes, les femmes font preuve d'attitudes plus favorables que les hommes et d'un désir d'accompagner leurs enfants jusqu'au bout dans leur cursus scolaire. D'après le ministère de l'éducation nationale (2012), les écoles où les mères sont bénéficiaires de l'allocation affichent un taux d'abandon inférieur de $30 \%$ à celles où les pères sont bénéficiaires. De même, le taux de réinscription des abandons est de $15 \%$ supérieur, quand les mères sont bénéficiaires. 
Tableau 4. Niveau d'instruction des parents et attitudes vis -à vis des modalités de subventions

\begin{tabular}{|l|l|c|c|}
\hline \multicolumn{2}{|c|}{} & \multicolumn{2}{|c|}{$\begin{array}{c}\text { Pensez-vous que les modalités de la subvention actuelle } \\
\text { encouragent les parents à envoyer les enfants à l'école ? }\end{array}$} \\
\cline { 2 - 4 } & Oui & Non \\
\hline \multirow{2}{*}{ Niveau } & Sans & $66,4 \%$ & $33,6 \%$ \\
d'instruction & Primaire & $58,2 \%$ & $41,8 \%$ \\
du chef de ménage & Secondaire & $60,4 \%$ & $39,6 \%$ \\
& Supérieur & $50,0 \%$ & $50,0 \%$ \\
\hline
\end{tabular}

Le tableau ci-dessus ne montre aucune relation significative entre le niveau d'instruction du chef de ménage et les attitudes favorables à l'égard des subventions destinées à encourager la scolarité des enfants. De plus, les parents dont le niveau d'instruction est faible ou quasiment nul témoignent d'une attitude favorable à l'égard de la scolarité des enfants et de leur engagement positif vis-à-vis de l'éducation. Ceci confirme les hypothèses qui postulent la tendance des parents ayant un niveau d'instruction faible à encourager leurs enfants à entreprendre un cursus scolaire avancé. Cette tendance s'explique par un alignement de comportements similaires à ceux adoptés par les familles intellectuelles. Autrement dit, les parents évoluent vers un encouragement à des études prolongées et vers une dépendance à l'école considérée comme une condition sine qua non de l'identité et de la réussite sociales.

\section{DISCUSSION DES RÉSULTATS}

Notre recherche a permis de tester la présence d'un altruisme intergénérationnel reflété par les attentes éducatives des familles marocaines pauvres, telles qu'elles apparaissent dans les déclarations des familles bénéficiant d'une subvention dans le cadre du programme Tayssir. Les résultats obtenus montrent des attitudes favorables au prolongement des études des enfants, et ce même en cas d'arrêt des subventions. De surcroît, le niveau d'instruction des parents ne semble pas affecter leurs attentes éducatives, dans la mesure où les parents les moins instruits sont tout aussi favorables au prolongement des études que ceux qui ont un niveau d'instruction élevé. D'autre part, l'arrêt des subventions ne déclenche pas un revirement des intentions des parents. En effet, même en cas d'arrêt, la quasi-totalité des parents, d'après leurs déclarations, continueront d'envoyer leurs enfants à l'école. En d'autres termes, l'aide financière est un moyen de soutenir les parents dans leur engagement et de réduire la lourdeur des investissements entrepris, et non d'influencer leur comportement. Enfin, l'étude a aussi révélé un engagement beaucoup plus important de la part des mères, qui sont plus disposées que les pères à investir et à utiliser au mieux les subventions. 
Il se peut que les familles ajustent leurs attentes en fonction de leur classe sociale. Ainsi, les parents, et plus précisément les mères, manifestent des aspirations élevées, afin de combler les frustrations subies ou d'éviter à leurs enfants les effets néfastes de la pauvreté. La réussite scolaire des enfants et leur insertion professionnelle ultérieure pourraient promouvoir socialement la famille entière.

Toutefois, il convient de signaler que le programme Tayssir est en phase d'expérimentation, et que rien ne garantit, jusqu'à présent, la généralisation du programme au niveau national. En cas d'abandon du programme, une nouvelle recherche pourrait porter sur la possible différence entre les déclarations et les actes des parents avant et après l'arrêt des subventions. D'autre part, le versement des subventions aux mères favorise la pérennité du programme. En cas de généralisation, cet élément devra être pris en compte.

\section{BIBLIOGRAPHIE}

DESLANDES R. (2009) : Family-school-community partnerships: What has been done? What have we learned? in R. Deslandes (ed.) International perspectives on contexts, communities and evaluated innovative practices (162-176), New York, N.Y.: Routledge.

EPSTEIN J.L. (2008) : School, family, and community partnerships: Preparing educators and improving schools. Your handbook for action (Third edition). Thousand Oaks, CA : Corwin Press.

FAN X. et CHEN M. (2001) : Parental involvement and students' academic achievement: A meta-analysis. Educational Psychology Review, 13:1-22.

JEYNES W.H. (2005) : A meta-analysis of the relation of parental involvement to urban elementary school student academic achievement. Urban Education, 40:237-69.

HENDERSON A. T., JOHNSON V., MAPP K. L. et DAVIES D. (2007) : Beyond the bake sale: The essential guide to family/school partnerships. New York, NY : New Press.

DIALLO K. (2001) : L'influence des facteurs familiaux, scolaires et individuels sur l'abandon scolaire des filles en milieu rural, de la région de Ségou (Mali). Thèse de doctorat (Ph.D.) Université de Montréal, spécialité sciences de l'éducation.

BEHRMAN J. \& SUSSANGKAN C. (1989) : Parental Schooling and Child Outcomes: Mother versus Father, Schooling Quality, and Interactions. Document polycopié. Philadelphie : University of Pennsylvania.

BIRDSALL N. (1980) : A Cost of Sibling: Child Schooling in Urban Colombia. Research in Population Economics, 2:115-50.

KING E. \& LILLARD L. (1987) : Determinants of Schooling Attainment and Enrollment Rates in the Phillipines. Santa Monica, CA : The Rand Corporation.

WOLFE B. \& BEHRMAN J. (1984): Determinants of Women's Health Status and Healthcare Utilization in a Development Country: A Latent Variable Approach. Review of Economics and Statistics, 56(4):696-703. 